\title{
Seis Sigma para la mejora continua en el mantenimiento de equipos pesados en empresas constructoras medianas.
}

\section{Six Sigma for constant improvement in the maintenance of heavy equipment in medium- sized construction companies.}

\author{
Manuel Zegarra ${ }^{1}$
}

http://dx.doi.org/10.21503/CienciayDesarrollo.2014.v17i2.02

\section{RESUMEN}

El objetivo principal de mantenimiento de los equipos de construcción es asegurar la operación de la máquina durante su ciclo de vida (vida útil de la inversión), permitiendo una alta disponibilidad y confiabilidad de la maquinaria relacionada. Estos objetivos se pueden lograr cuando los procesos de gestión de mantenimiento cuentan con una estructura administrativa adecuada y se siga el ciclo de la mejora continua que incluye planes, tareas, controles y acciones (los cuatro principios de la teoría de la administración) con procesos claramente establecidos y registro de datos en cada uno de esos procesos. La aplicación de la filosofía de la mejora continua usando el método Seis Sigma para la búsqueda de la excelencia como visión compartida y compromiso del cambio cultural es una herramienta administrativa de suma utilidad para analizar los indicadores clave de rendimiento (KPI) con el fin de tomar acciones; si fuera necesario para corregir los problemas antes de que causen daños graves. La gestión de mantenimiento será más eficiente y será posible manejar de una mejor manera los recursos con los que la empresa cuenta, usando los métodos administrativos para la mejora continua. El método Seis Sigma nos permitirá trabajar con sustento matemático y estadístico para tomar decisiones de mejora en los procesos que intervienen en la gestión de mantenimiento de equipos.

Palabras clave: Método Seis Sigma, filosofia Seis Sigma, mejora continua, gestión de mantenimiento.

\section{ABSTRACT}

The main objective of maintenance of construction equipment is to ensure the operation of the machine during its life cycle (life of the investment), enabling high availability and reliability of the related machinery. These objectives can be achieved when maintenance management processes have adequate administrative structure and the cycle of continuous improvement that includes plans, tasks, controls and actions (the four principles of management theory) processes remains clearly visible established and recording data in each of these processes. The application of the philosophy of continuous improvement using Six Sigma method for the pursuit of excellence as a shared vision and commitment of cultural change is an administrative tool very useful to analyze key performance indicators (KPI) to take actions; if necessary to correct problems before they cause serious damage. Maintenance management will be more

1 Doctor en Administración de la UAP, Maestro en Administración de la USMP, Ingeniero Mecánico de la PUCP, CIP 49526, Diplomado en ingeniería mecatrónica por la URP, especialista CAD-CAE por la PUCP, Black Belt Six Sigma por Caterpillar. Jefe de Monitoreo y Planeamiento en Ferreyros y asesor de empresas constructoras, docente del curso Gestión de Mantenimiento de equipo pesado en la PUCP. E-mail: mzegarraventura@hotmail.com; mzegarraventura@gmail.com 
efficient and be able to better manage the resources with which the company has, using administrative methods for continuous improvement. The Six Sigma method will allow us to work with mathematical and statistical decision making for improving the processes involved in managing equipment maintenance support.

Keywords: Method Six Sigma, Six Sigma philosophy, continuous improvement, maintenance management.

\section{INTRODUCCIÓN}

Dos sectores económicos importantes para las inversiones son el sector construcción y el sector minero. Las exigencias para poder ser sujeto de aprobación para la postulación en las licitaciones son grandes, por lo que la única forma que tienen las pequeñas y medianas empresas constructoras para poder desarrollarse dentro del sector y ser partícipes de estas inversiones, son dos: asociándose con empresas grandes o ser subcontratistas de las empresas ganadoras. Estas decisiones incluyen temas relativos a los equipos como adquisiciones, mantenimiento, reparaciones, reconstrucciones, eficiencias, disponibilidad, confiabilidad, reemplazos, costos y bajas de activos. Las empresas constructoras de nivel internacional están inmersas en lo que se denomina "El Mantenimiento de Clase Mundial", que significa tener políticas para realizar las labores cotidianas de trabajo con buenas prácticas tanto en el campo administrativo como en el campo técnico. La competitividad de las empresas constructoras reside en conseguir la máxima rentabilidad por metro cúbico movido o por la máxima cantidad de horas máquina vendida. En el primer aspecto la máxima rentabilidad depende de la máxima producción horaria y del mínimo costo horario de máquina. En el segundo aspecto la máxima rentabilidad depende de la mayor cantidad de horas trabajadas de la máquina y del menor costo horario de máquina. Por lo tanto, una adecuada gestión del mantenimiento del equipo mecánico, es importante para maximizar la rentabilidad empresarial, por lo que es necesario contar con herramientas que ayuden en este aspecto. La realidad en nuestro medio, muestra que el mantenimiento de equipos y máquinas en las empresas medianas y pequeñas de construcción está reducido a realizar cambios de aceites a las máquinas y en algunas se realiza un incipiente manejo administrativo de un programa preventivo básico. La mejora en la eficiencia de los procesos a fin de mantener o mejorar la disponibilidad y confiabilidad de los equipos, así como de los procesos del taller, será conseguida acogiendo la filosofía de la mejora continua, que es el espíritu de una visión de ir hacia la perfección. Usando el método administrativo Seis Sigma, ayudará al análisis de los resultados de la gestión de mantenimiento y permitirán la mejora continua, así como al alcance de los resultados esperados, traducidos en eficiencia en manejo del taller, mejora de la disponibilidad mecánica y aseguramiento de la vida útil de las máquinas. La presente investigación aporta conocimientos y un enfoque para mejorar la gestión de mantenimiento en las empresas constructoras medianas a fin de alcanzar la mayor eficiencia en los procesos y mejorar los resultados de gestión. El análisis se hizo sobre datos de los últimos 10 años de gestión de mantenimiento de una empresa constructora. La prueba piloto se realizó en una obra que duró 08 meses. En el trabajo de investigación se aplicó el proceso DMAIC del método Seis Sigma, incluyendo solo las etapas de DEFINIR, MEDIR, ANALIZAR y MEJORAR (DMAI) con establecimiento puntual de la mejora sugerida, sustentada con la comparación con una implementación piloto. La etapa de mejora no incluye lo referente a la 
implementación de las sugerencias en el proceso completo y tampoco se realizó la etapa de control, pues la implementación de las sugerencias depende de decisiones fuera del alcance del proyecto. Se toma el método administrativo Seis Sigma, como variable independiente, tomando como sus dimensiones la variabilidad de los procesos, el centrado de los procesos y los defectos por millón de oportunidades en los procesos. Se considera como variable dependiente a la mejora continua de la gestión de mantenimiento, tomando sus dimensiones la gestión de las labores del taller, la gestión de la disponibilidad operativa de las máquinas y la gestión de la probabilidad de cumplimiento de la vida útil de las máquinas. La investigación solo incluye lo relacionado a los procesos administrativos en el taller de mantenimiento. No se incluyen procesos referidos a operación de las máquinas, ni la programación de trabajos civiles en las obras. Se pretende con esta investigación aportar conocimientos acerca del uso del método Seis Sigma y de su filosofía en la mejora continua para la solución de problemas presentados en los procesos del mantenimiento mecánico y para la mejora de estos y su aplicación en empresas constructoras medianas ya que es un método con sustento matemático y estadístico que permite el análisis de eventos basado en datos de valores de tendencia central y de dispersión para obtener expresiones o proyecciones de resultados esperados dentro de límites de certeza e intervalos de confianza. Analizando los resultados de los subprocesos intervinientes, es posible relacionar estas curvas de probabilidad para pronosticar resultados futuros dentro de intervalos de confianza aceptables. Es un método moderno que es coherente con la administración científica y la clásica ya que se basa en análisis matemático para el aumento de productividad y para la mejora de los procesos.

\section{Problema principal}

¿De qué manera la aplicación del método Seis
Sigma ayudará en la mejora continua de la gestión de mantenimiento en las empresas constructoras medianas de Lima Metropolitana?

\section{Problemas secundarios}

1. ¿Cómo es que los defectos en el proceso afectan a la mejora continua de la gestión de las labores del taller en las empresas constructoras medianas de Lima metropolitana?

2. ¿De qué modo la variabilidad del proceso influye en la mejora continua de la disponibilidad operativa de la máquina en las empresas constructoras medianas de Lima Metropolitana?

3. ¿De qué forma el centrado del proceso incide en la mejora continua de la gestión de la probabilidad del cumplimiento de la vida útil de la máquina en las empresas constructoras medianas de Lima Metropolitana?

\section{Objetivo principal}

Analizar la relación entre la aplicación del método Seis Sigma y la mejora continua de la gestión de mantenimiento en las empresas constructoras medianas de Lima Metropolitana.

\section{Objetivos específicos}

1. Averiguar cómo afectan los defectos en el proceso a la mejora continua de la gestión de las labores del taller en las empresas constructoras medianas de Lima Metropolitana

2. Determinar cómo influye la variabilidad del proceso en la mejora continua de la gestión de la disponibilidad operativa de la máquina en las empresas constructoras medianas de Lima Metropolitana.

3. Identificar la manera en la cual el centrado del proceso incide en la mejora continua de la gestión de la probabilidad del cumplimiento de la vida útil de la máquina 
en las empresas constructoras medianas de Lima Metropolitana.

\section{Hipótesis general}

La aplicación del método Seis Sigma, ayuda en la mejora continua de la gestión de mantenimiento en las empresas constructoras medianas de Lima Metropolitana.

\section{Hipótesis secundarias}

1. Los defectos en el proceso afectan la mejora continua de la gestión de las labores del taller en las empresas constructoras medianas de Lima Metropolitana.

2. La variabilidad del proceso influye en la mejora continua de la gestión de la disponibilidad operativa de la máquina en las empresas constructoras medianas de Lima Metropolitana.

3. El centrado del proceso incide en la mejora continua de la gestión de la probabilidad del cumplimiento de la vida útil de la máquina en las empresas constructoras medianas de Lima Metropolitana.

\section{Identificación y clasificación de las variables e indicadores}

\section{Variable Independiente:}

El método Seis Sigma se basa en la medición y reducción de la variabilidad de los procesos que se analizan. Es por este motivo que se ha escogido como dimensión de esta variable a la desviación estándar del proceso analizar.

Estas dimensiones se medirán con los indicadores siguientes:

- Capacidad del proceso $(\mathrm{Cp}, \mathrm{Pp})=$

- Centrado del proceso $(\mathrm{CpK}, \mathrm{PpK}) \stackrel{L S E-L I E}{=} 6 \sigma$ $\min \left[\frac{L S E-\mu}{3 \sigma}\right.$ y $\left.\frac{\mu-L I E}{3 \sigma}\right]$ o La letra $\mathrm{K}$ viene del Japonés Katayori (かたより), que significa desvío o descentrado (American Society of Quality, 2013).

- Defectos por millón de oportunidades $($ DPMO $)=$ Cantidad de defectos en el producto por cada millón de oportunidades de defectos.

\section{Variable dependiente:}

Una buena labor en la gestión de mantenimiento se verá reflejada en los valores de las dimensiones siguientes:

- El tiempo que una máquina está en el campo produciendo.

- El tiempo que una máquina está inoperativa en reparación.

- Elmanejodel planeamientoyla programación de las reparaciones.

- La disponibilidad mecánica de la máquina.

- El cumplimiento de la vida útil de la máquina.

Estas dimensiones son medidas con los siguientes indicadores:

- TPEP (Tiempo promedio entre paradas o MTBS por las siglas en inglés)

- TPPR (Tiempo promedio para reparaciones o MTTR por las siglas en inglés)

- TPEF (Tiempo promedio entre fallas o MTBF por sus siglas en inglés)

- PRP (\% de reparaciones programadas sobre reparaciones totales)

- $\mathrm{DM}$ (Disponibilidad mecánica)

- R (Confiabilidad)

El indicador PRP o \%RP, muestra la buena o mala gestión del mantenimiento programado, su seguimiento, su ejecución y su calidad. El indicador TPPR es el tiempo promedio de demora de una reparación y refleja la efectividad de las reparaciones tanto en taller y campo. El indicador TPEP refleja el número de horas 
promedio que la máquina trabaja sin necesidad de paradas para intervenirla y es un indicador de la calidad de las reparaciones efectuadas y del buen uso que se le da al equipo. La disponibilidad mecánica es función del TPPR y del TPEP e indica la capacidad potencial de la máquina para estar trabajando y produciendo dinero. La confiabilidad o probabilidad del cumplimiento de la vida útil (probabilidad de vida útil sin fallas), es función del TPEF e indica la probabilidad de éxito en el cumplimiento de la vida útil del equipo en perfectas condiciones de operación.

\section{MATERIALES Y MÉTODOS}

La presente investigación es del tipo aplicada y cuasi-experimental, documental, retrospectiva $\mathrm{y}$ prospectiva. El nivel es correlacional y explicativo. El método es cuantitativo y se usó el método científico, se aplicaron los conocimientos de las ciencias administrativas y del mantenimiento de maquinaria y equipos, se usaron plantillas adecuadamente elaboradas de recolección de información de los indicadores de mantenimiento y se utilizaron los datos de una empresa constructora representativa del sector.

La población está constituida por empresas constructoras medianas de Lima Metropolitana.

Estas empresas tienen entre todas aproximadamente 2,000 máquinas medianas y grandes (entre 50 y $350 \mathrm{hp}$ de potencia Neta). La muestra está constituida por datos del historial de máquinas de la empresa TyT SAC Contratistas Generales que es una empresa constructora mediana de Lima Metropolitana que en conjunto posee 120 equipos de los cuales 58 máquinas están en los rangos de potencia entre 50 y $350 \mathrm{hp}$ de potencia neta. Se trata de un muestreo intencional u opinático.
El presente trabajo se realizó con limitaciones de presupuesto y personal calificado. Sin embargo la empresa colaboró en la mejora de los procesos para el piloto efectuado en la obra designada para hacer que la documentación llegue a tiempo para ser procesada y haciendo que el personal realice sus funciones de acuerdo a lo indicado en los manuales de funciones.

\section{Teoría del método Seis Sigma}

El método Seis Sigma postula la excelencia de los procesos y es por esta razón que se usa para reducir la variabilidad de éstos. El método Seis Sigma tiene una filosofía y un procedimiento. Como filosofía, postula la excelencia mediante la reducción de la variabilidad de los procesos y con la eliminación de los defectos, por lo que debe haber un cambio de mentalidad en el personal de la empresa, desde la más alta autoridad hasta el empleado de más bajo nivel, de tal manera que, bajo una visión compartida y que esta filosofía se encuentre internalizada en cada uno de los empleados, para que se puedan lograr los objetivos. Como proceso exige rigurosidad en su aplicación, siguiendo los pasos establecidos por el método. Una diferencia significativa entre Seis Sigma y similares programas en los años pasados, es el grado en el cual la administración juega un rol importante en los programas de monitoreo de resultados y cumplimiento de los objetivos (Pande, 2002, pág. 22). El método Seis Sigma tiene oportunidad de uso en todos los ámbitos de producción de bienes y servicios, pues:

- Donde hay producción (de bienes o servicios) hay procesos.

- En todos los procesos existe variabilidad.

- Donde hay variabilidad hay una oportunidad de mejora o disminución de esta variabilidad.

- Donde hay oportunidad de mejora de la variabilidad de los procesos, hay una oportunidad para la filosofía Seis Sigma. 


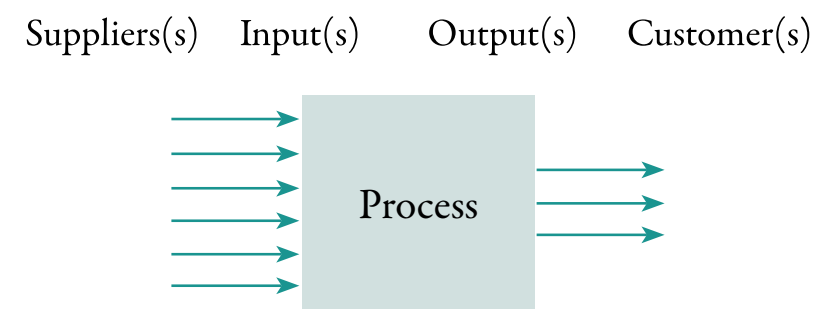

Grafico 1. Esquema del SIPOC (Caterpillar, 2011)

\section{Etapa DEFINIR}

El objetivo de la etapa DEFINIR es establecer la definición del propósito y alcance del proyecto y obtener información sobre antecedentes sobre el proceso y sus clientes (Brasard, 2005, pág. 12).

\section{CHARTER DEL PROYECTO}

\section{Caso de negocio (oportunidad de mejora) Declaración del problema (voz del cliente)}

La mejora de los procesos de gestión de Existe la necesidad de mejorar las condiciones de mantenimiento traerá beneficios a la empresa, las máquinas. Las máquinas se malogran con más por la mayor disposición de las máquinas en obra frecuencia de lo esperado.

al mejor costo de producción.

La alta frecuencia de paradas de equipos trae problemas.

Una baja disponibilidad operativa de maquina trae gastos no presupuestados como son los gastos administrativos por paralizaciones de obra, multas, y gastos por reposición de maquinaria mientras se repara las máquinas falladas.

\section{Declaración de metas}

Y1: Establecer la mejora en la gestión de taller

Y2: Establecer la mejora de la disponibilidad operativa

Y3: Establecer la mejora de la confiabilidad

Plan del proyecto

Definir:

Medir:

Analizar:

Mejorar:

Controlar

\section{Alcance del proyecto}

Incluye:

procesos de mantenimiento preventivo y predictivo, desde su programación hasta el control de los resultados.

No incluye:

procesos de mantenimiento correctivo ni diferentes a los mencionados arriba.

\section{Equipos seleccionado}

patrocinador: Gerente general, gerente de obras Dueño del proyecto: Jefe de equipos

Analisista: Mg Manuel Zegarra - BB Six Sigma

Asistente: Personal del área de mantenimiento de la empresa.

El CHARTER es un documento vivo que puede ser modificado en el transcurso del proyecto.

(Elaboración propia) 


\begin{tabular}{|c|c|c|c|c|}
\hline \multicolumn{5}{|c|}{$\begin{array}{c}\text { SIPOC DEL PROYECTO } \\
\text { TyT SAC contratistas generales }\end{array}$} \\
\hline Proveedor & Entradas & Proceso & Salidas & Cliente \\
\hline \multirow{15}{*}{$\begin{array}{l}\text { Sección } \\
\text { equipos } \\
\text { almacén taller }\end{array}$} & $\begin{array}{l}\text { Personal } \\
\text { mantenimiento }\end{array}$ & Plan de mantenimiento & \multirow{15}{*}{$\begin{array}{l}\text { Disponibilidad } \\
\text { mecanica en } \\
\text { campo } \\
\text { Bajo regimen de } \\
\text { paradas } \\
\text { Buena } \\
\text { condición de la } \\
\text { máquina }\end{array}$} & \multirow{15}{*}{$\begin{array}{l}\text { Obras propias } \\
\text { Obras } \\
\text { consorcios } \\
\text { Cliente } \\
\text { externos }\end{array}$} \\
\hline & Procesos & $\downarrow$ & & \\
\hline & $\begin{array}{l}\text { Software } \\
\text { Entrenamiento }\end{array}$ & Avance de Horómetros & & \\
\hline & Capacidad & $\downarrow$ & & \\
\hline & $\begin{array}{l}\text { técnica } \\
\text { Capacidad }\end{array}$ & Aviso de eventos & & \\
\hline & administrativa & $\downarrow$ & & \\
\hline & $\begin{array}{l}\text { Programación de } \\
\text { mantenimiento }\end{array}$ & Programación & & \\
\hline & Herramientas & $\downarrow$ & & \\
\hline & Instrumentos & Ejecución técnica & & \\
\hline & Manuales & $\downarrow$ & & \\
\hline & $\begin{array}{l}\text { Plan de } \\
\text { mantenimiento }\end{array}$ & Análisis resultados & & \\
\hline & Repuestos a & $\downarrow$ & & \\
\hline & $\begin{array}{l}\text { tiempo } \\
\text { Repuestos de } \\
\text { calidad }\end{array}$ & $\begin{array}{l}\text { Reparaciones } \\
\text { programadas }\end{array}$ & & \\
\hline & Infraestructura & & & \\
\hline & $\begin{array}{l}\text { Horómetros de } \\
\text { las máquinas }\end{array}$ & & & \\
\hline
\end{tabular}

\section{Grafico 2. SIPOC del proyecto}

Macro proceso TyT SAC

$\mathrm{Xs}, \mathrm{Ys}, \mathrm{Y}$

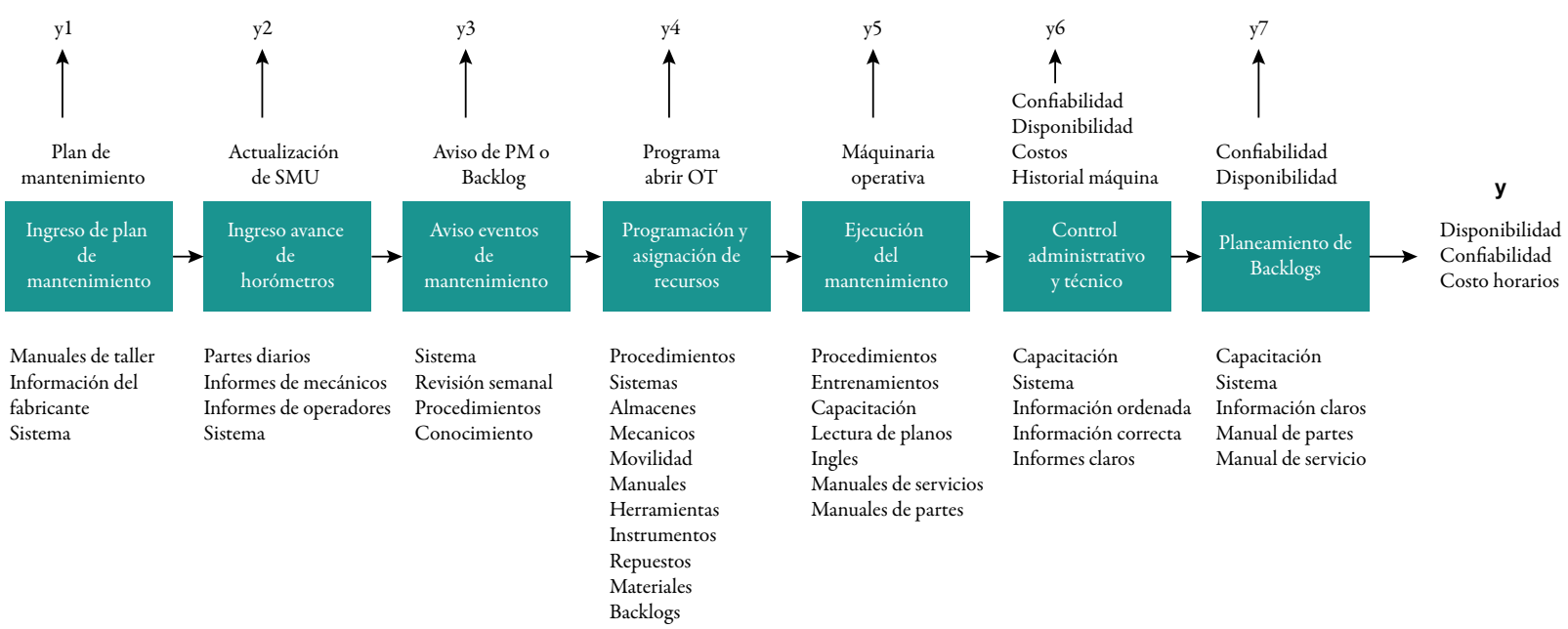

Grafico 3. Mapa del proceso (vista de 30000 pies) 


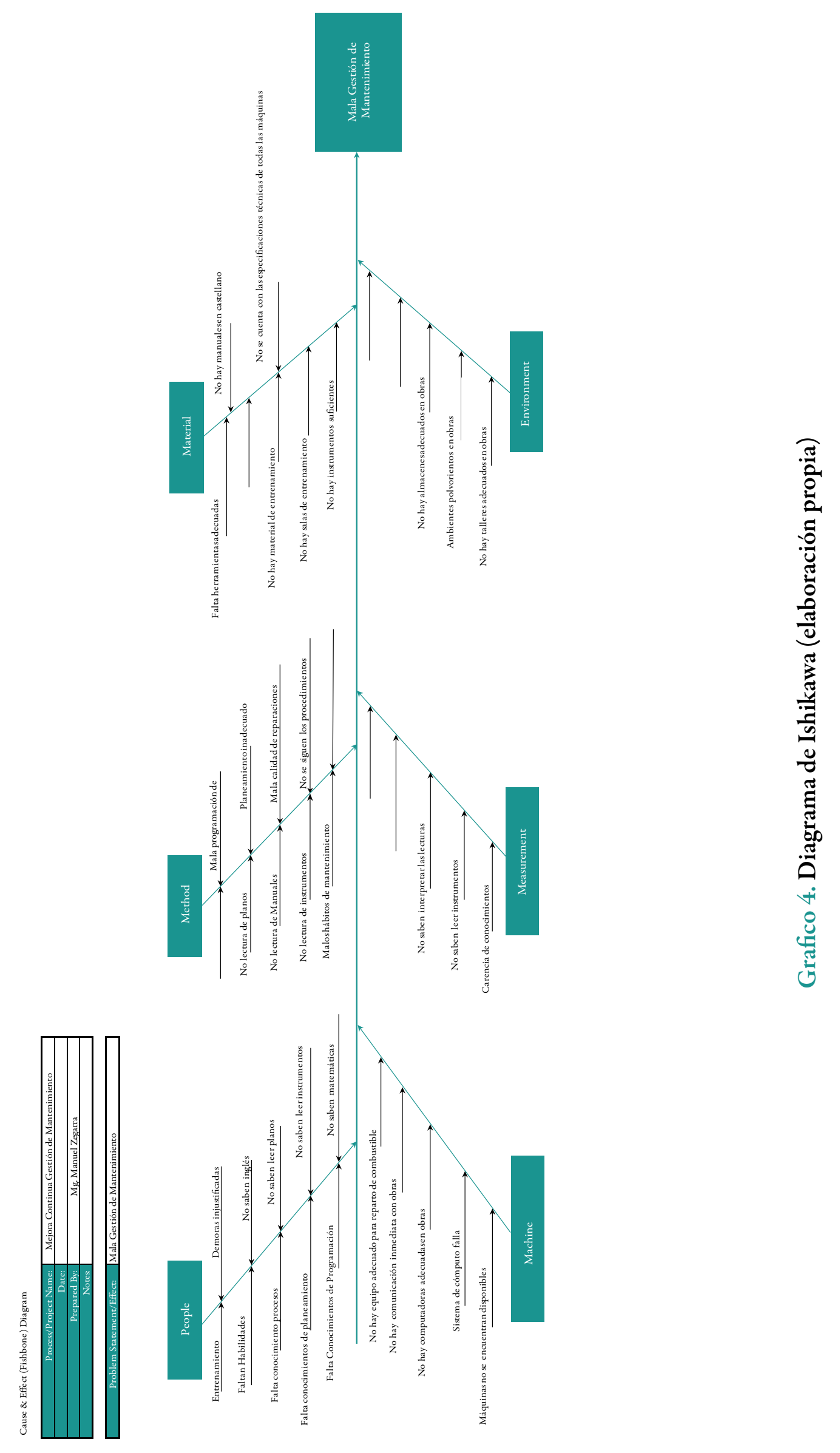




\section{Etapa MEDIR}

Durante la etapa MEDIR, se investiga el tema que se está estudiando, en detalle. Lo que efectivamente está sucediendo, cuándo sucede y dónde está sucediendo. Se reúnen datos, para establecer una línea base del desempeño, contra las que se compararán los resultados que se obtengan después que se trabaje el paso MEJORAR (Brasard, 2005, pág. 15). En esta etapa se midió para cada máquina el número de paradas, número de mantenimientos programados, horas trabajadas, número de órdenes de trabajo programadas, número de órdenes de trabajo no programadas, horas usadas en los mantenimientos.

\section{Etapa ANALIZAR}

El objetivo de la etapa es ANALIZAR e identificar CAUSAS RAÍZ y confirmarla con datos. La etapa ANALIZAR precisa las causas específicas de la declaración enfocada del problema que se desarrolla como resultado del paso MEDIR (Brasard, 2005, pág. 18).

Tabla 1

\begin{tabular}{|c|c|c|c|c|c|}
\hline $\begin{array}{l}\text { Dimensión } \\
\text { de la variable } \\
\text { dependiente }\end{array}$ & $\begin{array}{c}\text { Indicador: } \\
\text { Variable } \\
\text { independiente }\end{array}$ & $\begin{array}{c}\text { Dimensión } \\
\text { de la variable } \\
\text { independiente }\end{array}$ & $\begin{array}{l}\text { Indicador: variable } \\
\text { independiente }\end{array}$ & $\begin{array}{l}\text { Resultado encontrado } \\
\text { en el proceso (total de } \\
\text { equipos y máquinas) } \\
\text { (antes) }\end{array}$ & $\begin{array}{c}\text { Resultado } \\
\text { encontrado en } \\
\text { el proceso (clase } \\
\text { máquinas) (antes) }\end{array}$ \\
\hline \multirow{4}{*}{$\begin{array}{l}\text { Gestión de las } \\
\text { labores de taller }\end{array}$} & $\% \mathrm{RP}$ & \multirow{4}{*}{$\begin{array}{c}\text { Defectos en el } \\
\text { proceso }\end{array}$} & DPMO (\%error) & $379,300(37.93 \%)$ & $447,400(44.74 \%)$ \\
\hline & TPEP о MTBS & & DPMO (\%error) & $862,100(86.21 \%)$ & $815,800(81.58 \%)$ \\
\hline & TPPR & & DPMO (\%error) & $\begin{array}{l}500,000(50 \%) \\
293,100<\text { LIE } \\
206,900>\text { LSE }\end{array}$ & $\begin{array}{l}500,000(50 \%) \\
263,200<\text { LIE } \\
236,800>\text { LSE }\end{array}$ \\
\hline & TPEF о MTBF & & DPMO (\%error) & $482,800(48.28 \%)$ & $394,700(39.74 \%)$ \\
\hline $\begin{array}{c}\text { Gestión de la } \\
\text { disponibilidad } \\
\text { operativa }\end{array}$ & $\mathrm{DM}$ & $\begin{array}{l}\text { Variabilidad del } \\
\text { proceso }\end{array}$ & Pp o Cp & 0.91 & 1.01 \\
\hline $\begin{array}{l}\text { Gestión de la } \\
\text { probabilidad de } \\
\text { cumplimiento } \\
\text { de la vida útil de } \\
\text { la máquina }\end{array}$ & Confiabilidad & $\begin{array}{l}\text { Centrado del } \\
\text { proceso }\end{array}$ & PpK o CpK & 0.11 & 0.26 \\
\hline
\end{tabular}

Los valores de los resultados nos muestran que existe una gran oportunidad para mejorar los procesos. Como mencionamos anteriormente, es necesario tener claro que llegar a los niveles Seis Sigma requiere de mucho esfuerzo, mucho trabajo en equipo, fuerte decisión de la gerencia y directores de la empresa y normalmente una gran inversión, que todas las empresas no están dispuestas a hacer, salvo que el mercado se los justifique. Un comienzo simple y no oneroso es el de comprometer al personal de la empresa a seguir los procedimientos establecidos de manera rigurosa. En tal sentido se hicieron talleres de lluvia de ideas con el personal involucrado en los procesos del mantenimiento y se obtuvo el siguiente diagrama de Ishikawa, que sirvió para elaborar la matriz de análisis de modo de fallas y efectos (Failure Mode and Effects Analysis FMEA). Esta matriz nos muestra los potenciales modos de falla, sus efectos probables, los riesgos de ocurrencia de las fallas y las acciones que debemos tomar para corregir los problemas. 


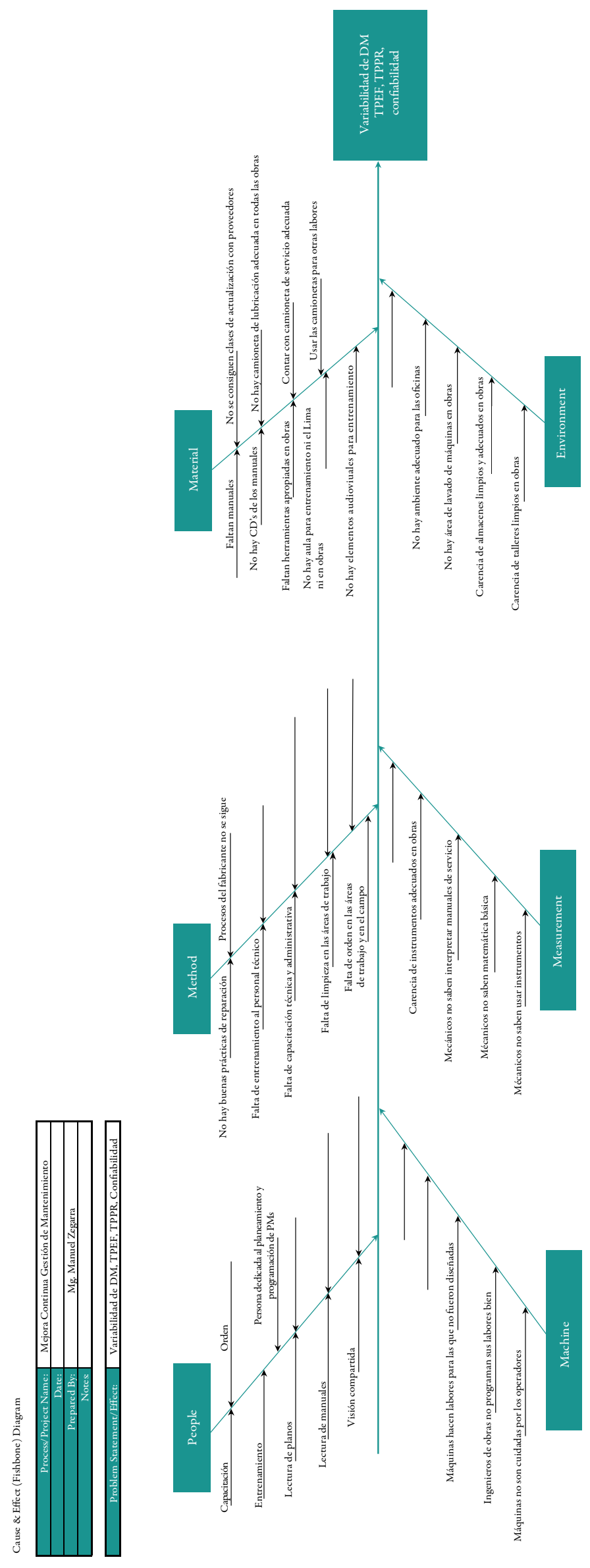




\section{Tabla 2. Matriz FMEA (elaboración propia)}

\section{Potential Failure Mode \& Effects Analysis}

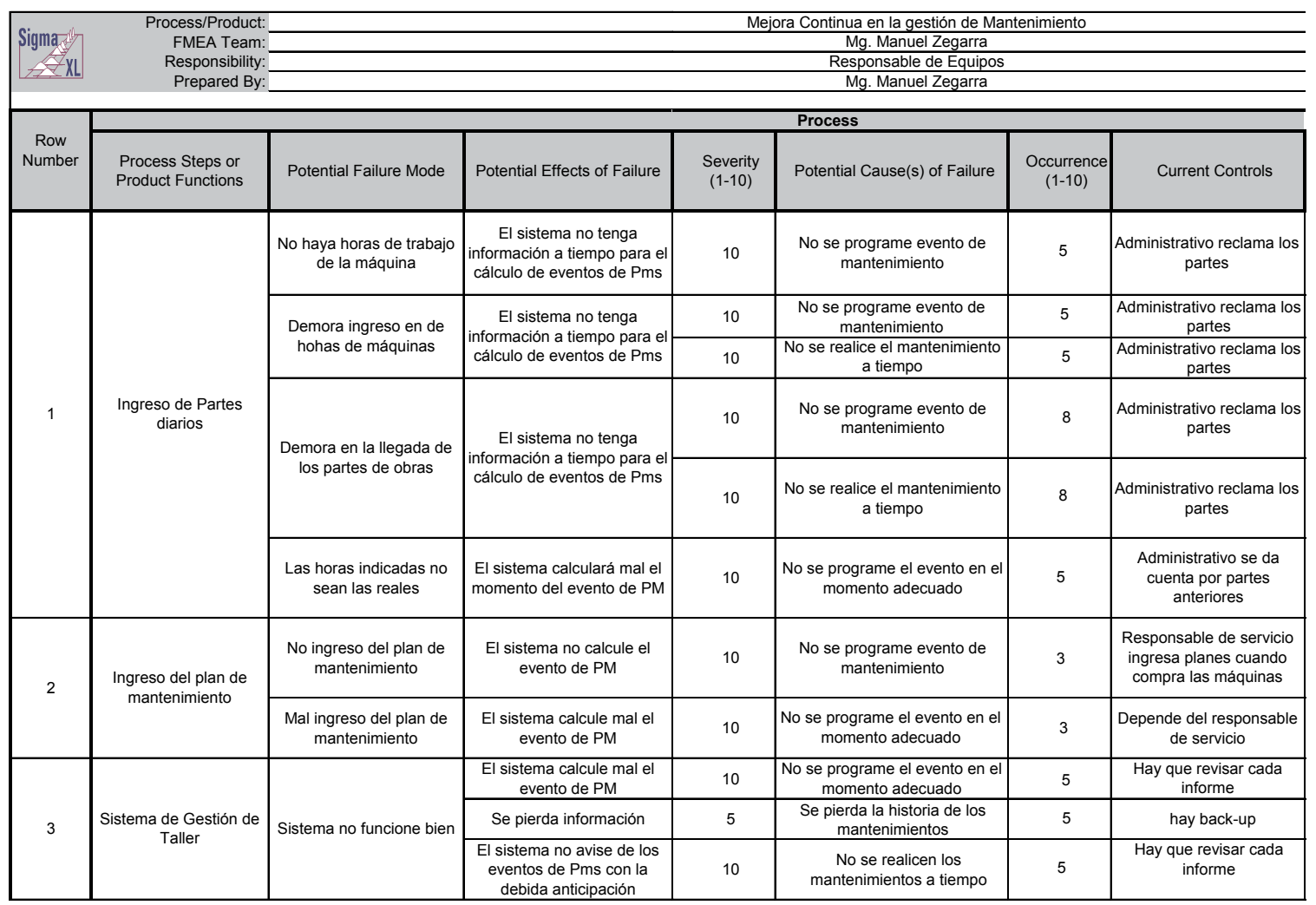

\begin{tabular}{|c|c|c|c|c|c|c|c|}
\hline \multirow{9}{*}{4} & \multirow{9}{*}{ Asignación de recursos } & $\begin{array}{c}\text { No haya repuestos en } \\
\text { almacén }\end{array}$ & $\begin{array}{l}\text { No se disponga de los } \\
\text { repuestos a tiempo }\end{array}$ & 10 & $\begin{array}{l}\text { No se realice el mantenimiento } \\
\text { a tiempo }\end{array}$ & 3 & Hay estock de protección \\
\hline & & $\begin{array}{c}\text { Falta capacidad de Mano } \\
\text { de Obra }\end{array}$ & $\begin{array}{c}\text { No haya mecanicos } \\
\text { disponibles para el } \\
\text { mantenimiento }\end{array}$ & 10 & $\begin{array}{l}\text { No se realice el mantenimiento } \\
\text { a tiempo }\end{array}$ & 4 & $\begin{array}{l}\text { hay que programar con } \\
\text { tiempo }\end{array}$ \\
\hline & & \multirow{2}{*}{$\begin{array}{l}\text { Falta cantidad adecuada } \\
\text { de instrumentos } y \\
\text { herramientas }\end{array}$} & \multirow{2}{*}{$\begin{array}{l}\text { No se cuente con } \\
\text { instrumentos y herramientas } \\
\text { al momento de los PMs }\end{array}$} & 10 & $\begin{array}{l}\text { Se realice un mal serivicio de } \\
\text { mantenimiento }\end{array}$ & 5 & no \\
\hline & & & & 10 & $\begin{array}{l}\text { La máquina vuelva a fallar ren } \\
\text { el corto tiempo }\end{array}$ & 5 & no \\
\hline & & \multirow{3}{*}{$\begin{array}{l}\text { Falta cantidad adecuada } \\
\text { de manuales y planos }\end{array}$} & \multirow{2}{*}{$\begin{array}{c}\text { No se cuente con los } \\
\text { manuales y planos } \\
\text { necesarios para realizar el } \\
\text { mantenimiento }\end{array}$} & 10 & $\begin{array}{l}\text { Se realice un servicio de } \\
\text { mantenimiento de mala calidad }\end{array}$ & 5 & no \\
\hline & & & & 10 & $\begin{array}{l}\text { La máquina vuelva a fallar ren } \\
\text { el corto tiempo }\end{array}$ & 5 & no \\
\hline & & & $\begin{array}{l}\text { No se ingrese el plan de } \\
\text { mantenimiento correcto }\end{array}$ & 10 & $\begin{array}{l}\text { No se realice el mantenimiento } \\
\text { en el momento adecuado }\end{array}$ & 3 & no \\
\hline & & $\begin{array}{c}\text { No hay unidades de } \\
\text { mantenimiento adecuadas }\end{array}$ & $\begin{array}{l}\text { No se llegue a tiempo al } \\
\text { mantenimiento }\end{array}$ & 10 & $\begin{array}{l}\text { Máquina parada } \\
\text { innecesariamente }\end{array}$ & 6 & no \\
\hline & & $\begin{array}{c}\text { Las unidades son } \\
\text { utilizadas en otras labores }\end{array}$ & $\begin{array}{l}\text { No se llegue a tiempo al } \\
\text { mantenimiento }\end{array}$ & 10 & $\begin{array}{l}\text { Máquina parada } \\
\text { innecesariamente }\end{array}$ & 6 & no \\
\hline \multirow{6}{*}{5} & \multirow{6}{*}{$\begin{array}{l}\text { Entrenamiento y } \\
\text { capacitación técnica }\end{array}$} & \multirow{5}{*}{$\begin{array}{l}\text { No se realizan las clases } \\
\text { de entrenamiento }\end{array}$} & \multirow{3}{*}{$\begin{array}{l}\text { Mala capacitación de los } \\
\text { mecánicos }\end{array}$} & 10 & $\begin{array}{c}\text { Se realice un mal } \\
\text { mantenimiento, con demora }\end{array}$ & 7 & no \\
\hline & & & & 10 & $\begin{array}{l}\text { Demora en la realización del } \\
\text { mantenimiento }\end{array}$ & 7 & no \\
\hline & & & & 10 & $\begin{array}{l}\text { Máquina vuelve a fallar en el } \\
\text { corto tiempo }\end{array}$ & 7 & no \\
\hline & & & \multirow{2}{*}{$\begin{array}{c}\text { Mala interpretación de los } \\
\text { manuales y lectura de planos }\end{array}$} & 10 & $\begin{array}{c}\text { Se realice un mal } \\
\text { mantenimiento, con demora }\end{array}$ & 5 & no \\
\hline & & & & 10 & $\begin{array}{l}\text { Máquina vuelve a fallar en el } \\
\text { corto tiempo }\end{array}$ & 5 & no \\
\hline & & $\begin{array}{c}\text { Carencia de sala de } \\
\text { entrenamiento y equipos } \\
\text { audiovisuales }\end{array}$ & $\begin{array}{l}\text { No se realizan los } \\
\text { entrenamientos }\end{array}$ & 10 & Mecánicos mal entrenados & 7 & no \\
\hline 6 & $\begin{array}{l}\text { Alimentación de } \\
\text { combustible }\end{array}$ & Camión no adecuado & Despacho de petroleo sucio & 10 & Máquina va a fallar & 7 & no \\
\hline
\end{tabular}


Estas mejoras no requieren inversión adicional de parte de la empresa, pues el personal de ella que labora en las diferentes áreas puede trabajar en ellas como parte de la mejora continua. Un análisis de las causas de los problemas presentados, nos lleva a las siguientes sugerencias para la mejora de los procesos:

- Es importante el constante mantenimiento del software del sistema integrado de mantenimiento.

- Revisión del algoritmo del software, para un adecuado cálculo de los mantenimientos por venir y de los cálculos de la información que se puede obtener, como son los costos horarios, horas trabajadas por las máquinas en las obras, consumos horarios de combustible, aceites, repuestos, etc.

- Envío oportuno de los documentos de taller, a la oficina central. Incluye los partes diarios de operación de las máquinas, reportes de fallas e informes de mantenimiento, etc.

- Ingreso oportuno de los datos en el software del sistema de gestión del mantenimiento.

- Verificación de la información a ingresar, en caso se sospeche de alguna inconsistencia. La corrección debe ser inmediata.

- Verificación de los planes de mantenimiento de las máquinas.

- Ingresodeinformacióndelosmantenimientos realizados.

- Manejo adecuado de los mantenimientos programados.

- Manejo adecuado de los backlogs o reparaciones programadas (no cambios de aceite programados).

- Implementar ciclos de capacitación técnica más seguidos, para los mecánicos y para los administrativos. Los técnicos deben recibir clases y capacitación en las buenas prácticas de mantenimiento y en la parte administrativa que les corresponde. Los administrativos, deben tener entrenamiento en lo correspondiente al ingreso de datos y también lo básico en la parte técnica para que puedan estar atentos cuando haya un dato inconsistente en la información que viene de obras. Se recomienda la práctica de las clases semanales a los mecánicos donde se discuten problemas presentados y cómo se solucionaron, así como concretar con los proveedores de máquinas el dictado de clases técnicas según los modelos de máquinas que el taller tenga. Estas clases deben ser desarrolladas por el responsable de equipos de cada taller (Lima o provincias).

- Contratar mecánicos e ingenieros calificados para cuando haya necesidad de ello, por ejemplo para las obras.

- Revisión de la cantidad y calidad de los instrumentos y herramientas necesarios para poder realizar los mantenimientos y las reparaciones.

- Asegurar los procedimientos de atención de almacén, para que provean a tiempo de los repuestos necesarios para los mantenimientos y las reparaciones.

- Seguir estrictamentelos procesos establecidos con revisiones bimensuales de cumplimiento de objetivos relativos al porcentaje de reparaciones programadas, tiempos de las reparaciones y calidad de las mismas.

\section{Etapa MEJORAR}

El objetivo de esta etapa es desarrollar, probar e implementar soluciones que ataquen las causa raíz y utilizar datos para evaluar las soluciones como también los planes que se utilizarán para realizarla (Brasard, 2005, pág. 19).

Las salidas de esta etapa son:

- Acciones planeadas y probadas que eliminan o reducen el impacto de $\mathrm{la}(\mathrm{s})$ causa(s) identificada(s) de un problema.

- Análisis de los datos "Antes" y "Después", que demuestren qué tanto se cerró la brecha inicial. Esto es comparar los resultados del piloto con los resultados de la línea base.

- Una comparación del plan en relación con la implementación real. 
En esta etapa corresponde hacer un piloto (experimento controlado) para la validación y sustento de las recomendaciones. Para este efecto, se implementó un plan de ejecución y seguimiento de los procedimientos que la empresa ya tiene establecidos, pero se puso esmero y cuidado en seguirlos de manera rigurosa.

A continuación se presentan los resultados comparadas con las líneas base (antes).
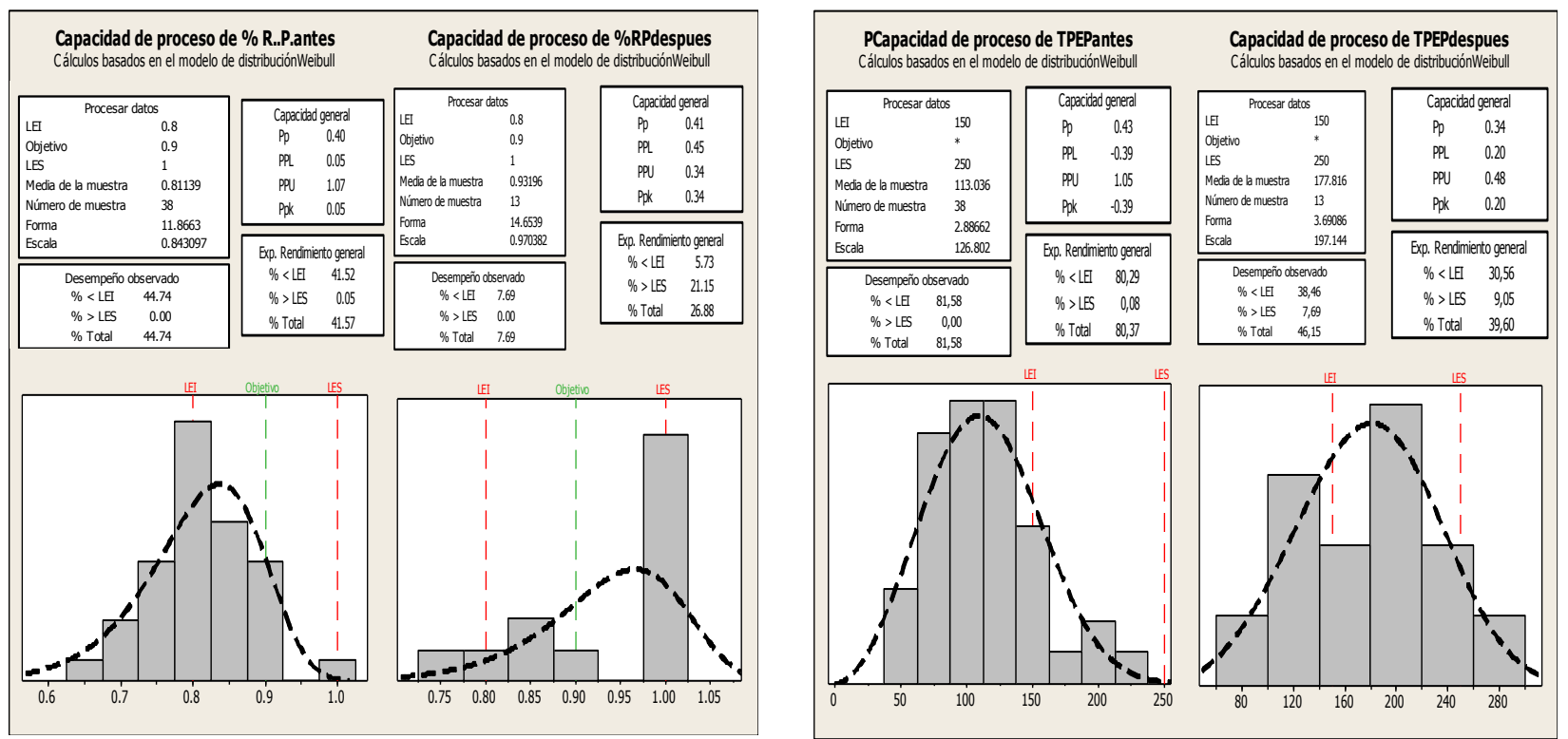

Gráfico 6. Capacidad de proceso del \% RP \& TPEP antes y después (elaboración propia)
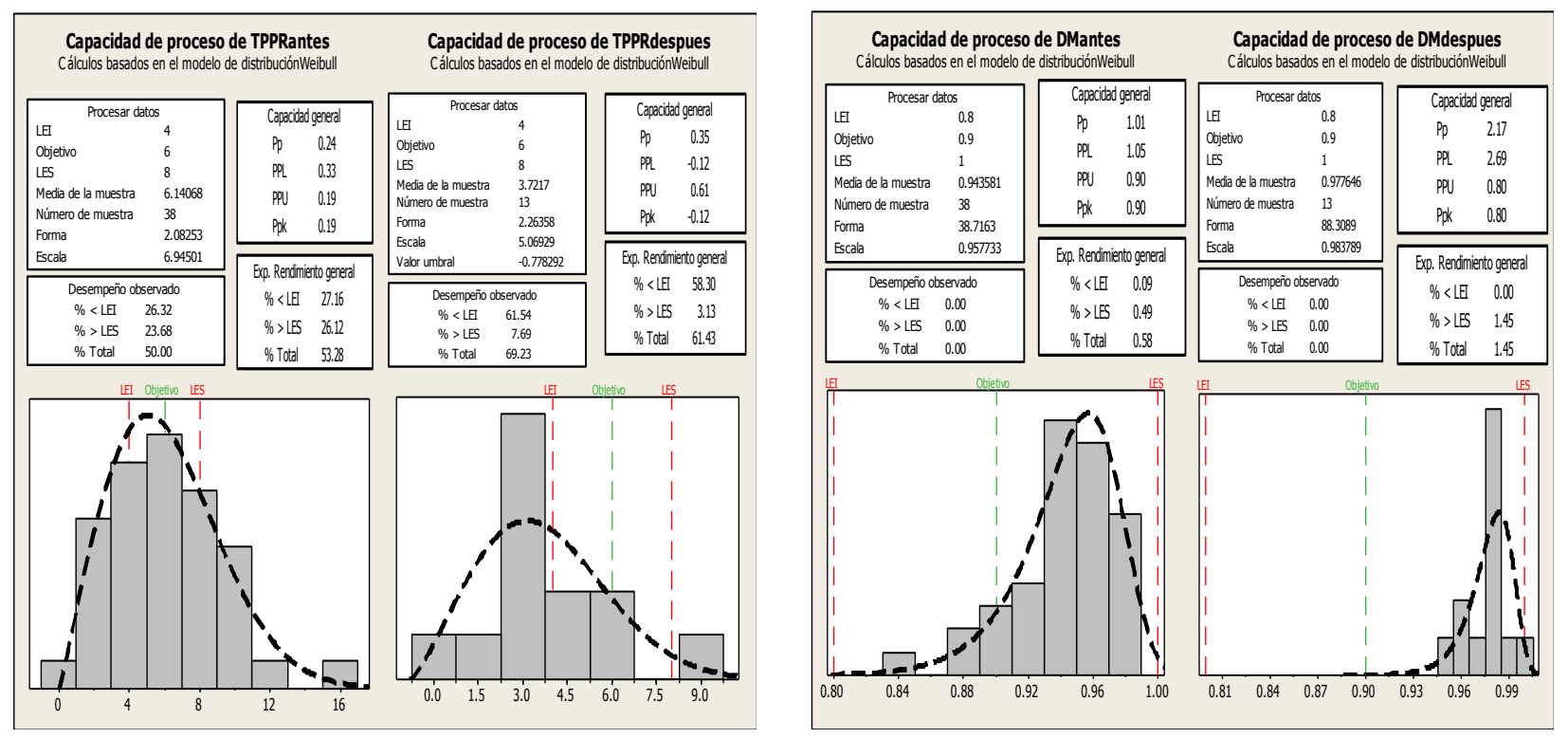

Gráfico 7. Capacidad de proceso del TPPR y DM antes y después (elaboración propia) 

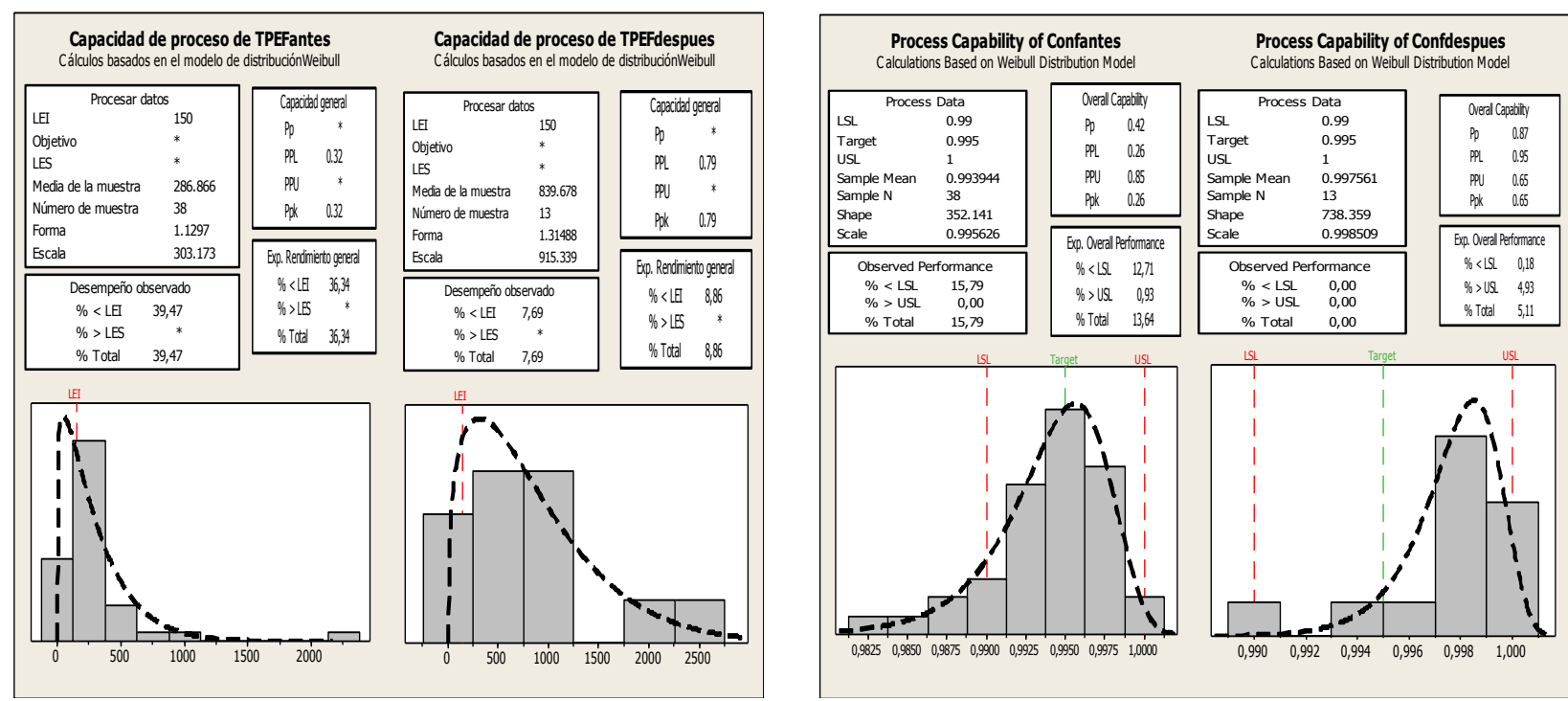

Gráfico 8. Capacidad de proceso del TPEF y $R$ antes y después (elaboración propia)

La tabla siguiente presenta el resumen de los valores p-value de las pruebas $\mathrm{t}$-student pareadas para la prueba de hipótesis.

\begin{tabular}{cccc} 
Variable & pValue & Rechazo/aceptación Ho & $\begin{array}{c}\text { Muestras estadísticamente } \\
\text { diferentes }\end{array}$ \\
\hline \% RP & $0.1 \%$ & Rechazo & $\mathrm{Si}$ \\
\hline TPEP & $0.1 \%$ & Rechazo & $\mathrm{Si}$ \\
\hline TPPR & $0.5 \%$ & Rechazo & $\mathrm{Si}$ \\
\hline DM & $0 \%$ & Rechazo & $\mathrm{Si}$ \\
\hline TPEF & $1.6 \%$ & Rechazo & $\mathrm{Si}$ \\
\hline $\mathrm{R}$ & $0.1 \%$ & Rechazo & $\mathrm{Si}$ \\
\hline
\end{tabular}

Donde:

$\mathrm{H} 0=$ Las medias son iguales (hipótesis nula) $\mathrm{Ha}=$ Las medias son diferentes (hipótesis alterna)

pValue $>0.05$ (5\%), significa que se acepta $\mathrm{H} 0$ pValue $<0.05$ (5\%), significa que se rechaza $\mathrm{HO}$ y se acepta $\mathrm{Ha}$
En las siguientes páginas mostramos los cuadros de comparación de las capacidades de trabajo, los valores de porcentaje de defectos y los índices de centrado de los procesos antes y después. Se observa una mejora importante en los todos los indicadores. 


\section{Tabla 3. Resumen resultados finales (elaboración propia)}

\begin{tabular}{|c|c|c|c|c|c|c|}
\hline $\begin{array}{l}\text { Dimensión } \\
\text { de la variable } \\
\text { dependiente }\end{array}$ & $\begin{array}{c}\text { Indicador: } \\
\text { Variable } \\
\text { independiente }\end{array}$ & $\begin{array}{c}\text { Dimensión } \\
\text { de la variable } \\
\text { independiente }\end{array}$ & $\begin{array}{l}\text { Indicador: variable } \\
\text { independiente }\end{array}$ & $\begin{array}{c}\text { Resultado encontrado } \\
\text { en el proceso (total de } \\
\text { equipos y máquinas) } \\
\text { (antes) }\end{array}$ & $\begin{array}{c}\text { Resultado } \\
\text { encontrado en } \\
\text { el proceso (clase } \\
\text { máquinas) (antes) }\end{array}$ & $\begin{array}{l}\text { Resultados del } \\
\text { piloto (despues) }\end{array}$ \\
\hline \multirow{4}{*}{$\begin{array}{l}\text { Gestión de las } \\
\text { labores de taller }\end{array}$} & $\% \mathrm{RP}$ & \multirow{4}{*}{$\begin{array}{c}\text { Defectos en el } \\
\text { proceso }\end{array}$} & DPMO (\%error) & $379,300(37.93 \%)$ & $447,400(44.74 \%)$ & $78,900(7.87 \%)$ \\
\hline & TPEP о MTBS & & DPMO (\%error) & $862,100(86.21 \%)$ & $815,800(81.58 \%)$ & $\begin{array}{c}461,500 \\
(46.15 \%)\end{array}$ \\
\hline & TPPR & & DPMO (\%error) & $\begin{array}{l}500,000(50 \%) \\
293,100<\text { LIE } \\
206,900>\text { LSE }\end{array}$ & $\begin{array}{l}500,000(50 \%) \\
263,200<\mathrm{LIE} \\
236,800>\text { LSE }\end{array}$ & $\begin{array}{c}692,300 \\
(69.23 \%) \\
615,400<\text { LIE } \\
76,900>\text { LSE }\end{array}$ \\
\hline & TPEF o MTBF & & DPMO (\%error) & $482,800(48.28 \%)$ & $394,700(39.74 \%)$ & $76,900(7.69 \%)$ \\
\hline $\begin{array}{l}\text { Gestión de la } \\
\text { disponibilidad } \\
\text { operativa }\end{array}$ & $\mathrm{DM}$ & $\begin{array}{c}\text { Variabilidad del } \\
\text { proceso }\end{array}$ & Pp o Cp & 0.91 & 1.01 & 2.17 \\
\hline $\begin{array}{l}\text { Gestión de la } \\
\text { probabilidad de } \\
\text { cumplimiento } \\
\text { de la vida útil de } \\
\text { la máquina }\end{array}$ & Confiabilidad & $\begin{array}{c}\text { Centrado del } \\
\text { proceso }\end{array}$ & PpK o CpK & 0.11 & 0.26 & 0.65 \\
\hline
\end{tabular}

La implementación completa de la MEJORA, va a quedar bajo la decisión del dueño del proyecto y de los dueños de los procesos, ya que esta requiere de modificaciones o mejora en el seguimiento de los procesos internos de la empresa y la necesaria colaboración de otras áreas.

Process Management -

Involvement by 6 Sigma Role

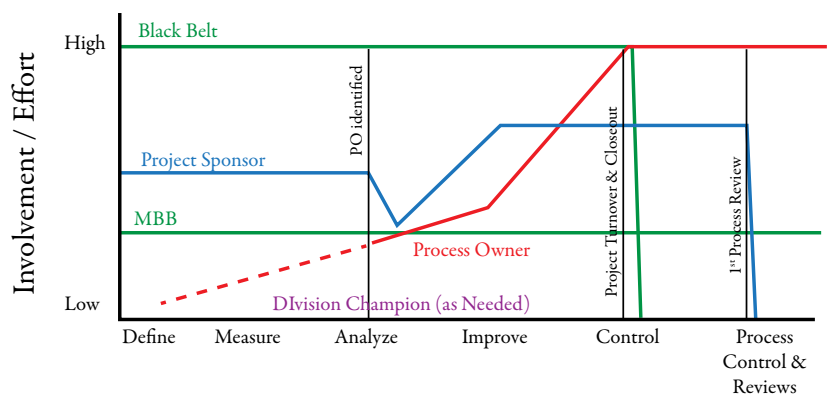

Gráfico 9. Participación de los actores en el método seis sigma (Brasard, 2005)

\section{Etapa CONTROLAR}

El objetivo del paso CONTROLAR es mantener los logros que se han obtenido, mediante la estandarización de los métodos, de trabajo y procesos, anticipando mejoramientos futuros y preservando las lecciones que se hayan aprendido (Brasard, 2005, pág. 22). 


\section{CONCLUSIONES}

1. La aplicación del método Seis Sigma, ayuda en la mejora continua de la gestión de mantenimiento de la empresa TyT SAC Contratistas Generales y contribuirá en la mejora continua de la gestión de mantenimiento de las pequeñas y medianas empresas constructoras de Lima Metropolitana.

2. La aplicación del método Seis Sigma contribuye para detectar y disminuir los defectos en el proceso afectando positivamente a la mejora continua de la gestión de las labores del taller de la empresa TyT SAC Contratistas Generales.

3. La aplicación del método Seis Sigma contribuye en la mejora de la variabilidad del proceso influenciando positivamente en la mejora continua de la gestión de disponibilidad operativa de la máquina en la empresa TyT SAC Contratistas generales.

4. La aplicación del método Seis Sigma ayuda a controlar el centrado del proceso incidiendo de manera positiva en la mejora continua de la gestión de la probabilidad de cumplimiento de la vida útil de la máquina de la empresa TyT SAC Contratistas Generales.

\section{Trabajos citados}

1. American Society of Quality. (2013). Introduction to quality engineering. ASQ.

2. Brasard, M. (2005). El impulsor de la memoria II, Seis Sigma (Primera ed.). USA: GOAL/ QPC.

3. Brassard, M. (2002). The Six Sigma Memory Jogger II (Primera ed.). USA: GOAL/QPC.

4. Chiavenato, I. (1999). Introducción a lateoría general de la administración (Quinta ed.). McGraw-Hill.

5. Chiavenato, I. (2006). Introducción a la teoría general de la administración (Séptima ed.). McGraw-Hill.f

6. Koontz, H., \& Weihrich, H. (2004). Administración, una perspectiva global (Duodécima ed.). McGraw-Hill.

7. Zegarra, M. (2007). La administración como herramienta de gestión para optimizar el mantenimiento mecánico en la empresa TyT $S A C$ Contratistas Generales. Lima, Lima, Lima: Tesis inédita. 